10,11

\title{
Особенности перколяционной схемы перестройки колебательного спектра сплава с составом для $\mathrm{Ga}_{1-x} \mathrm{Al}_{x} \mathrm{P}$
}

\author{
(C) С.П. Козырев \\ Физический институт им. П.Н. Лебедева РАН, \\ Москва, Россия \\ E-mail: skozyrev@sci.lebedev.ru \\ (Поступила в Редакцию 30 октября 2017 г.)
}

\begin{abstract}
Рассмотрены особенности свойств решеточных колебаний $\mathrm{Ga}-\mathrm{P}$ в рамках перколяционной модели смешанного кристалла (сплава) $\mathrm{Ga}_{1-x} \mathrm{Al}_{x} \mathrm{P}$ с нулевым решеточным рассогласованием между бинарными компонентами сплава. В отличие от других двухмодовых систем сплавов, в $\mathrm{Ga}_{1-x} \mathrm{Al}_{x} \mathrm{P}$ перколяционное расщепление $\delta \sim 13 \mathrm{~cm}^{-1}$ наблюдается для низкочастотной моды $\mathrm{GaP-подобных} \mathrm{колебаний.} \mathrm{Отщепленная}$ от основной, дополнительная GaP-мода (одна из компонент перколяционного дуплета) проявляется в сплаве, обогащенном $\mathrm{GaP}$, и совпадает по частоте со щелью с нулевой плотностью однофононных состояний кристалла GaP. Также представлен расчет колебательного спектра примесного $\mathrm{Al}$ в кристалле $\mathrm{GaP}$ с использованием теории динамики кристаллической решетки. При замещении атома $\mathrm{Ga}$ более легким $\mathrm{Al}$ помимо локальной моды, в рассчитанном спектре в области щели с нулевой плотностью фононных состояний кристалла $\mathrm{GaP}$ наблюдается особенность, совпадающая с экспериментально наблюдаемой модой на частоте $378 \mathrm{~cm}^{-1}$ в сплаве $\mathrm{Ga}_{1-x} \mathrm{Al}_{x} \mathrm{P}(x<0.4)$.
\end{abstract}

DOI: 10.21883/FTT.2018.04.45693.306

\section{1. Введение}

Перколяционная модель полупроводникового сплава (твердого раствора), основанная на теории перколяции (the percolation site theory) [1], была предложена Pagès atal. [2] для интерпретации особенностей спектра комбинационного рассеяния света (КРС) сплавов халькогенидов (Zn, Be)VI (VI соответствует элементу VI группы) - в области BeVI-подобных колебаний наблюдается дуплет сильных колебательных мод с большим расщеплением. Замещение атомов $\mathrm{Zn}$ атомами Ве с малым ковалентным радиусом приводит к локальному уменьшению длины связи с соответствующим увеличением ее жесткости и значительному топологическому разупорядочению, вызванному таким замещением. Идея перколяционной модели заключается в том, что топологическое разупорядочение приводит к композитному характеру твердого раствора $(\mathrm{Zn}, \mathrm{Be}) \mathrm{VI}$ из жестких (обогащенных Ве) и мягких (обогащенных $\mathrm{Zn}$ ) областей. Для жесткой связи Be-VI локальное окружение в этих двух областях сплава разное, разные силовые константы связи и соответственно частоты собственных колебаний. Для пары BeVI-подобных колебаний величина расщепления перколяционного дуплета составляет $\delta_{\mathrm{BeSe}} \sim 50 \mathrm{~cm}^{-1}$ в $(\mathrm{ZnBe}) \mathrm{Se}$ и $\delta_{\mathrm{BeTe}} \sim 40 \mathrm{~cm}^{-1}$ в $(\mathrm{ZnBe}) \mathrm{Te}$. Перколяционную схему $(1$-связь $\rightarrow 2$-моды $)$ перестройки спектра решеточных колебаний сплава с составом, предложенную для сплава (Zn, Be)VI с большим контрастом в жесткости связи бинарных компонент сплава, Pagès at al. [3] считают возможной для применения при интерпретации спектра решеточных колебаний сплавов $A_{1-x} B_{x} C$ с мягкими связями и соответственно с малым контрастом в жесткости, какими явля- ются типичные полупроводниковые сплавы. В нашей работе [4] была представлена попытка интерпретации некоторых особенностей спектра решеточных колебаний сплава $\mathrm{Cd}_{1-y} \mathrm{Hg}_{y}$ Те исходя из перколяционной модели сплава $A_{1-x} B_{x} C$ как композитной среды, образованной в результате естественных флуктуации в составе сплава на локальном уровне двумя сосуществующими $A C$ - и $B C$-обогащенными областями. Сплав $\mathrm{Cd}_{1-y} \mathrm{Hg}_{y} \mathrm{Te}$ образован полупроводником СdTe и полуметаллом $\mathrm{HgTe}$ с одинаковой структурой цинковой обманки (ZB) и почти равными решеточными параметрами $\left(a_{0}=6.482\right.$ и $6.461 \AA$ соответственно для СdТе и $\mathrm{HgTe})$. Из-за очень малого решеточного рассогласования бинарных компонент в сплаве $\mathrm{Cd}_{1-y} \mathrm{Hg}_{y}$ Те при катионном замещении длина связи с Те-фнионом не изменяется вне зависимости от того, находится ли катион в HgТе-обогащенной области или в CdTe-обогащенной области сплава. Это означает, что рассмотренная Pagès at al. [2,3] перколяционная схема перестройки колебательного спектра сплава с составом для $\mathrm{Cd}_{1-y} \mathrm{Hg}_{y}$ Те не применима. В их схеме (1-связь $\rightarrow 2$-моды) перколяционное расщепление в сплаве $A_{1-x} B_{x} C$ достигается за счет того, что и для $A-C$-связи, и для $B-C$-связи из-за различия решеточных параметров компонент сплава локальное окружение в $A C$ - и $B C$-обогащенных областях деформировано по-разному, что приводит к расщеплению частоты колебаний для данной связи. И чем больше решеточное рассогласование между компонентами сплава $A C$ и $B C$, тем больше величина расщепления перколяционного дуплета в сплаве. Подобные утверждения справедливы и для $A-C$-связи, и для $B-C$-связи, но для связи, соответствующей низкочастотным колебаниям, расщепление перколяционного дуплета значительно 

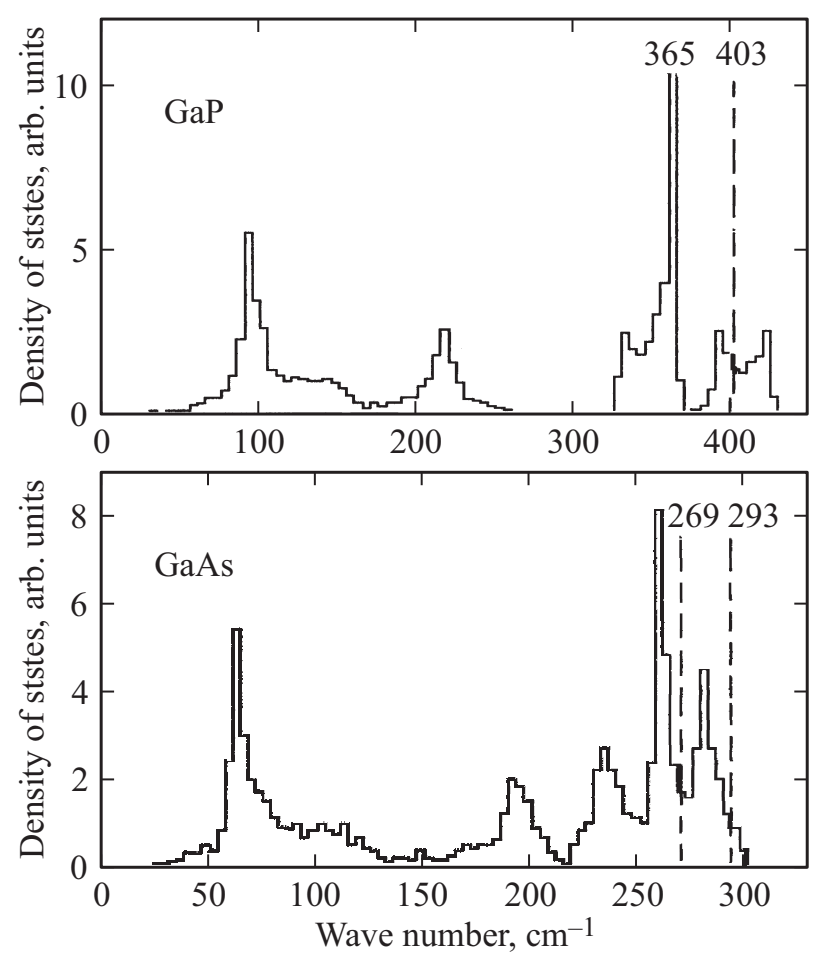

Рис. 1. a) Однофононная плотность состояний для $\mathrm{GaP}$ [10]. В спектральной области LO-TO-расщепления $\left(403-365 \mathrm{~cm}^{-1}\right)$ наблюдается щель с нулевой плотностью состояний. $b$ ) Однофононная плотность состояний для $\mathrm{GaAs}$ [10]. В спектральной области LO-TO расщепления $\left(293-269 \mathrm{~cm}^{-1}\right)$ щель с нулевой плотностью состояний отсутствует.

меньше и, как правило, спектрально не разрешается. В сплаве $\mathrm{Cd}_{1-y} \mathrm{Hg}_{y} \mathrm{Te}$ перколяционное расщепление $\delta_{\mathrm{HgTe}} \sim 16 \mathrm{~cm}^{-1}$ наблюдается для низкочастотной HgТе-подобной моды колебаний, которая ниже частоты CdTe-подобных колебаний. Интерпретация колебательного спектра в сплаве $\mathrm{Cd}_{1-y} \mathrm{Hg}_{y}$ Те допускает для атома $\mathrm{Hg}$ наличие двух решеточных состояний - c одноямным потенциалом в СdTе-обогащенной области сплава и с двуямным потенциалом в $\mathrm{HgTe-обогащенной}$ области [5]. Моды HgТе-подобных колебаний в этих областях соответствуют перколяционному дуплету. Проявление композитного характера сплава $\mathrm{Cd}_{1-y} \mathrm{Hg}_{y} \mathrm{Te}$ через наличие дополнительной отщепленной моды стало возможным благодаря различию типа химической связи в бинарных компонентах сплава: в СdTе связь считается ионно-ковалентной и осуществляется через $s p^{3}$-орбитали, а в $\mathrm{HgTe}$ - связь тяжелой $\mathrm{Hg}$ c Те в значительной степени формируется с участием $d$-орбиталей $[6,7]$.

Сплавы GaAlP и GaAlAsi, как и сплав CdHgTe, образованы бинарными компонентами с одинаковой структурой ZB и с почти нулевым решеточным рассогласованием. Для сплава GaAlP разница длин связи в бинарных компонентах GaP и AlP равна 2.360-2.367 = -0.007 для сплава GaAlAs разница длин связи в компонентах GaAs и AlAs равна 2.448-2.451 = -0.003 $\AA$. Оба сплава GaAlP и GaAlAs характеризуются одинаковым типом связи - преимущественно ковалентным, как и для других III-V-соединений. При равенстве длин связи катионные места в сплавах GaAlP и GaAlAs эквивалентны, и следовало бы ожидать, что спектр решеточных колебаний в сплаве любого состава будет характеризоваться только двумя основными колебательными модами бинарных компонент сплава. Из ранних пионерских исследований спектров решеточных колебаний в сплавах $\mathrm{Ga}_{1-x} \mathrm{Al}_{x} \mathrm{P}$ [8] и $\mathrm{Ga}_{1-x} \mathrm{Al}_{x} \mathrm{As}$ [9] установлено, что они соответствуют двухмодовому типу перестройки колебательного спектра с составом сплава. B отличие от сплава GaAlAs, где четко проявляются только две основные моды во всем интервале составов и никаких дополнительных структур, в сплаве GaAlP для составов с GaP-обогащением наблюдается дополнительная мода, отщепленная от основной $\mathrm{GaP}$-подобной моды. Наличие дополнительной структуры у низкочастотной GaP-моды при практическом отсутствии решеточного рассогласования бинарных компонент сплава делают спектр решеточных колебаний сплава $\mathrm{Ga}_{1-x} \mathrm{Al}_{x} \mathrm{P}$ подобным спектру сплава $\mathrm{Hg}_{1-x} \mathrm{Cd}_{x} \mathrm{Te}$ (индекс состава $1-x=y)$, для которого перколяционная схема перестройки колебательного спектра с составом сплава

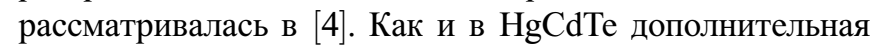
мода с $\omega=378 \mathrm{~cm}^{-1}$ в спектре решеточных колебаний сплава $\mathrm{Ga}_{1-x} \mathrm{Al}_{x} \mathrm{P}$ при малых $x$ попадает в щель с нулевой плотностью фононных состояний кристалла $\mathrm{GaP}$. На рис. $1, a$ и $1, b$ представлены однофононные плотности состояний для $\mathrm{GaP}$ [10] и для GaAs [10] соответственно. В плотности состояний для $\mathrm{GaP}$ щель с нулевой плотностью состояний расположена между LO $\left(=403 \mathrm{~cm}^{-1}\right)$ и ТО $\left(=365 \mathrm{~cm}^{-1}\right)$ модами колебаний $\mathrm{GaP}$. $\mathrm{B}$ перколяционной схеме перестройки колебательного спектра сплава дополнительная мода является следствием проявления композитного состава сплава. Она явля-

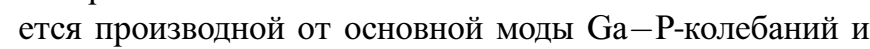
одной из компонент перколяционного дуплета. В плотности фононных состояний для GaAs (рис. $1, b$ ) щель с нулевой плотностью состояний в области частот между LO- и TO-модами отсутствует, и в спектре GaAlAs нет отщепленной моды.

\section{2. Особенности спектра решеточных колебаний в $\mathrm{Ga}_{1-x} \mathrm{Al}_{x} \mathrm{P}$}

O существовании дополнительной третьей моды с $\omega=378 \mathrm{~cm}^{-1}$, помимо основных мод GaP- и AlР-подобных колебаний, в двухмодовом сплаве $\mathrm{Ga}_{1-x} \mathrm{Al}_{x} \mathrm{P}$ сообщалось в ранней работе [11]. Для состава $x=0.2$ были даже представлены параметры трех осцилляторов (частота, сила осциллятора, параметр затухания), используемых при дисперсионном анализе экспериментального спектра решеточного отражения. И в сплаве $\mathrm{GaP}_{1-y} \mathrm{As}_{y}$ 
$(y=0.25)$ была обнаружена та же дополнительная мода c $\omega=378 \mathrm{~cm}^{-1}$. В сплаве $\mathrm{Ga}_{1-x} \mathrm{Al}_{x} \mathrm{As}$ эта мода отсутствует. В последующей работе [8] авторы исследовали для сплава $\mathrm{Ga}_{1-x} \mathrm{Al}_{x} \mathrm{P}$ композиционную зависимость частот двух мод GaP- и AlP-подобных колебаний, игнорируя возможное наличие дополнительной третьей моды, считая ее индуцированной разупорядочением LO-модой вблизи границы зоны Бриллюэна. Измерения спектров КРС [12] подтвердили наличие двух полос продольных LO-колебаний, приписываемых модам GaP- и AlP-подобных колебаний.

Исходя из результатов исследований работ, выполненных G. Lucovsci at al. [8,11], мы провели комбинированные измерения решеточного ИК-отражения и КРС на эпитаксиальных пленках $\mathrm{Ga}_{1-x} \mathrm{Al}_{x} \mathrm{P}$. Измерения проводились на пленках толщиной $6 \mu \mathrm{m}$, выращенных на подложке (111) GaP методом жидкофазной эпитаксии. Для исключения доминирующих в спектре КРС полос TO- и LO-фононов подложки GaP, наблюдаемых при нормальном падении возбуждающего света, спектры КРС возбуждались сфокусированным пучком лазера до размера $2-3 \mu \mathrm{m} \mathrm{c} \mathrm{торца} \mathrm{эпитаксиальной} \mathrm{пленки.} \mathrm{Ре-}$ зультаты дисперсионного анализа спектров решеточного отражения - концентрационная зависимость частот ТО-ветвей - были подтверждены данными анализа спектров КРС. В колебательных спектрах исследованных составов проявляются 3 моды GaР-подобных колебаний и одна мода AlP-подобных колебаний (например, для состава $x=0.43$ рис. $2, c)$. Из трех мод GaP-подобных колебаний одна мода с $\omega=365 \mathrm{~cm}^{-1}$ - мода $\mathrm{Ga}-\mathrm{P}$ колебаний бинарной компоненты сплава, другая мода с $\omega=378 \mathrm{~cm}^{-1}$ наблюдалась в [11], третья мода с $\omega=355 \mathrm{~cm}^{-1}-$ неизвестной природы, но ее наличие подтверждалось данными ИК- и КРС-измерений. Природа последних двух мод для нас оставалась неизвестной.

В настоящей работе мы заново провели интерпретацию спектров решеточного отражения сплава $\mathrm{Ga}_{1-x} \mathrm{Al}_{x} \mathrm{P}$, представленных ранее в [8]. Измерения в [8] проводились на пленках, полученных жидкофазной эпитаксией на подложку $\mathrm{GaP}$, с толщиной пленки до $100 \mu \mathrm{m}$, достаточной чтобы в отношении ИК-отражения в области решеточных колебаний исключить интерференцию света на границе раздела пленки и подложки и рассматривать пленки как объемный материал. Именно по этой причине, чтобы избежать неоднозначности в интерпретации $R(\omega)$, мы отказались от дальнейшей работы с тонкими пленками из [13]. Последующий дисперсионный анализ диэлектрической функции $\varepsilon(\omega)$ проводился представлением ее в аддитивной форме

$$
\varepsilon(\omega)=\varepsilon_{\infty}+\sum_{j} \frac{S_{j} \omega_{t j}^{2}}{\omega_{t j}^{2}-\omega^{2}-i \omega \gamma_{j}}
$$

Здесь $\omega_{t j}, S_{j}$ и $\gamma_{j}$ - частота $j$-ой решеточной ТО-моды, ее сила осциллятора и параметр затухания. Результаты дисперсионного анализа (частоты колебательных мод и
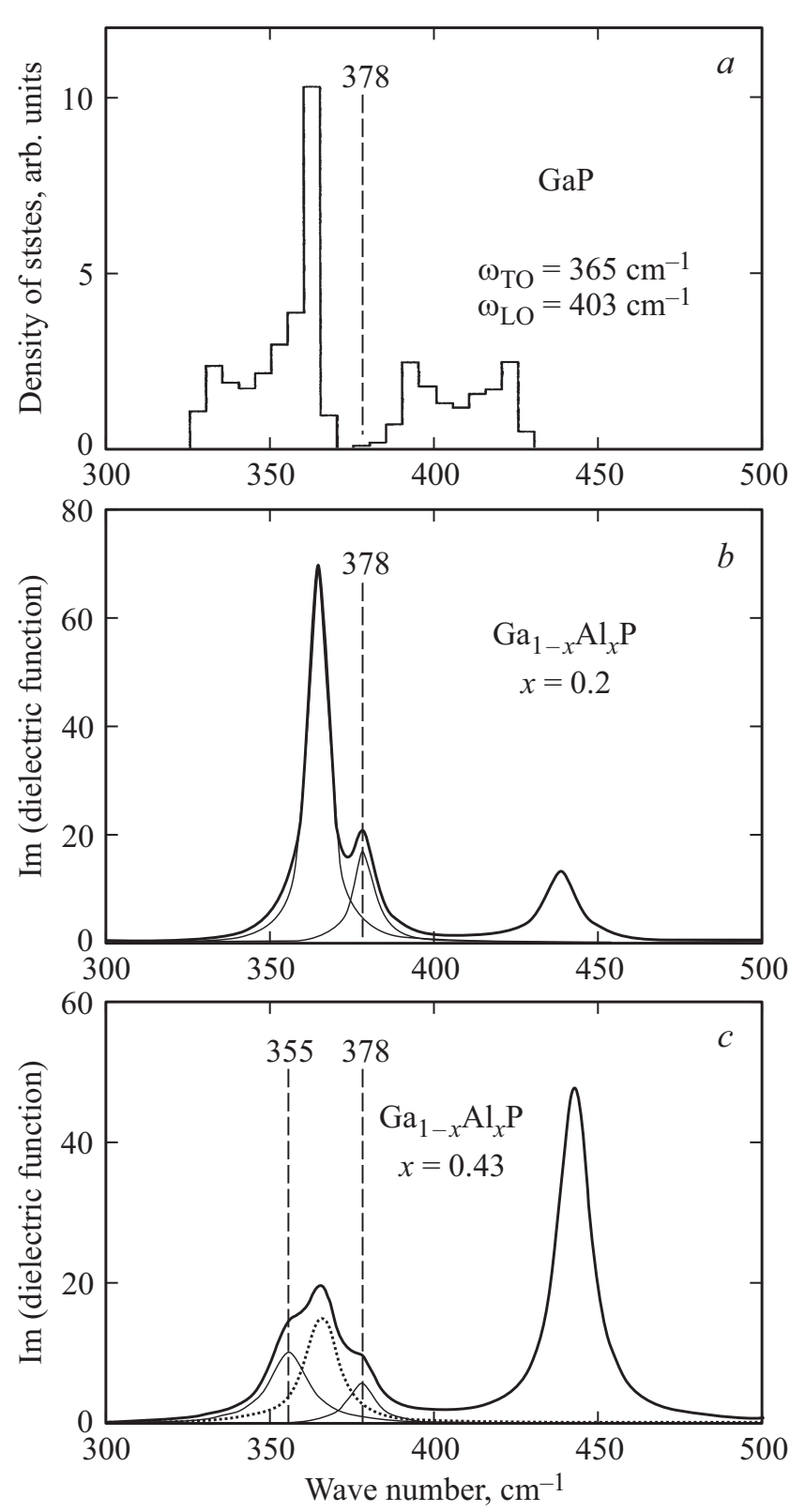

Рис. 2. a) Фрагмент функции однофононной плотности состояний в $\mathrm{GaP} . b)$ Мнимая часть диэлектрической функции $\operatorname{Im} \varepsilon(\omega)$ кристалла $\mathrm{GaP}$ с примесью $\mathrm{Al}(x=0.2)$, рассчитанная в соответствии с микроскопической теорией [14]. ТО мода с $\omega_{\text {Tо }}=365 \mathrm{~cm}^{-1}$ априорно задается при расчете. Особенность $\operatorname{Im} \varepsilon(\omega)$ на $\omega=378 \mathrm{~cm}^{-1}$ соответствует нулевой плотности фононных состояний в $\mathrm{GaP} . c$ ) Мнимая часть диэлектрической функции $\operatorname{Im} \varepsilon(\omega)$ сплава $\mathrm{Ga}_{1-x} \mathrm{Al}_{x} P \quad(x=0.43)$. В области $\mathrm{GaP-подобных} \mathrm{колебаний} \mathrm{при} x>0.4$ наблюдается дополнительная мода на $\omega=355 \mathrm{~cm}^{-1}$.

их силы осциллятора) для всего интервала составов сплава $\mathrm{Ga}_{1-x} \mathrm{Al}_{x} \mathrm{P}$ представлены на рис. 3. Распределения частот ТО-мод колебаний в сплаве в зависимости от состава (рис. 3,a) аналогичны представленным ранее в [13] для тонких пленок - одна мода $\mathrm{TO}_{\mathrm{Al}}$ AlP-подобных колебаний и три моды в области GaP-подобных 

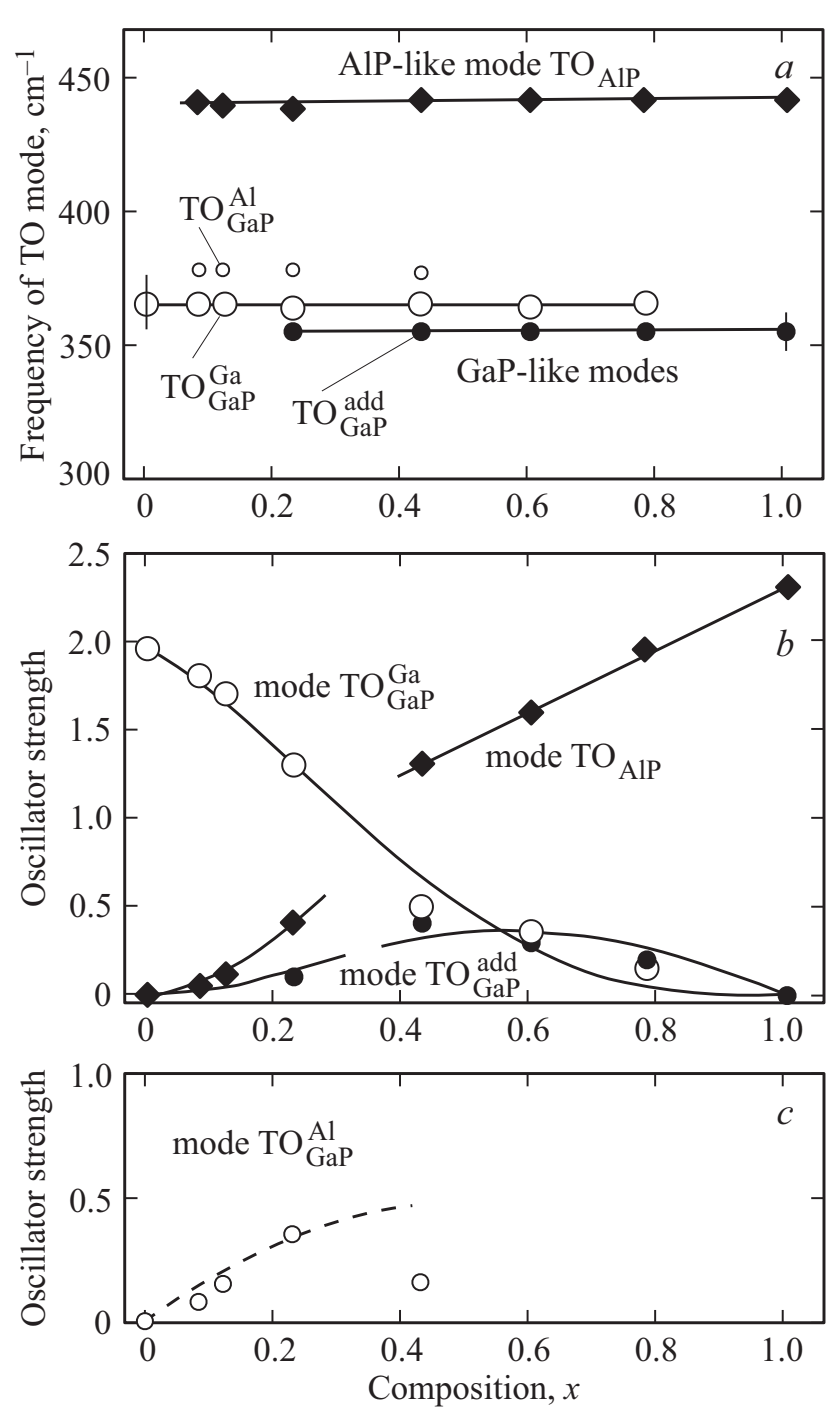

Рис. 3. а) Распределения частот $\mathrm{TO}_{\mathrm{GaP}}$ мод $\mathrm{GaP-подобных} \mathrm{ко-}$ лебаний и $\mathrm{TO}_{\mathrm{AlP}}$ моды AlР-подобных колебаний в зависимости от состава $x$ сплава $\mathrm{Ga}_{1-x} \mathrm{Al}_{x} \mathrm{P}$. Мода $\mathrm{TO}_{\mathrm{GaP}}^{\mathrm{Al}}$ - отщепленная от основной $\mathrm{TO}_{\mathrm{GaP}}^{\mathrm{Ga}}$ моды $\mathrm{GaP}$-подобных колебаний, вместе образуют перколяционный дуплет. $b$ ) Распределение сил осциллятора

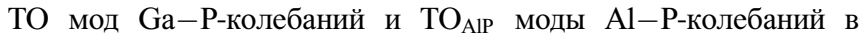
зависимости от состава $x$ сплава $\mathrm{Ga}_{1-x} \mathrm{Al}_{x} \mathrm{P}$. Распределение для моды $\mathrm{TO}_{\mathrm{GaP}}^{\mathrm{Ga}}$ с частотой $\omega \approx 365 \mathrm{~cm}^{-1}$ аппроксимируется расчетной кривой $S(x)=1.95\left[(1-x)^{4}+3 x(1-x)^{3}\right]$. c) Распределение силы осциллятора колебаний отщепленной моды $\mathrm{TO}_{\mathrm{GaP}}^{\mathrm{Al}}$ в зависимости от состава сплава.

колебаний. На рис. $3, b$ и 3,c представлены распределения сил осциллятора для соответствующих мод. Мода с $\omega=378 \mathrm{~cm}^{-1}\left(\mathrm{TO}_{\mathrm{Ga}}^{\mathrm{Al}}\right)$ проявляется для составов $x<0.5$, также как и в КРС-спектрах [13], причем ее интенсивность резко падает при $x \sim 0.3$ (рис. 3,c). При этом же составе $(x \sim 0.3)$ проявляется еще одна дополнительная мода $\mathrm{TO}_{\mathrm{Ga}}^{\mathrm{add}}$. На рис. $2, c$ представлена $\operatorname{Im} \varepsilon(\omega)$ для сплава $\mathrm{Ga}_{1-x} \mathrm{Al}_{x} \mathrm{P}(x=0.43)$. В области частот $\mathrm{GaP}$ подобных колебаний разрешаются три моды, одна из них $\mathrm{TO}_{\mathrm{GaP}}^{\mathrm{add}}$ с $\omega=355 \mathrm{~cm}^{-1}$, ее интенсивность сравнима c основной модой GaP-подобных колебаний $\mathrm{TO}_{\mathrm{GaP}}^{\mathrm{Ga}} \mathrm{c}$ $\omega=365 \mathrm{~cm}^{-1}$ для составов $x>0.4$ (рис. $3, b$ ). В сплаве составов $x \sim 0.4$ происходит структурная модификация. На рис. 4 представлена суммарная сила осцилляторов $S_{\mathrm{GaP}}$ для всех $\mathrm{GaP}$-подобных колебаний в зависимости от состава сплава $x$ (показано темными символами) совместно с распределением сил осциллятора $S_{\mathrm{AlP}}$ для AlPподобных колебаний. При рассмотрении изменения состава сплава со стороны AlP (от $x=1)$ суммарная сила осцилляторов $S_{\mathrm{GaP}}$ для $\mathrm{GaP}$-подобных колебаний линейно увеличивается с составом сплава $S_{\mathrm{GaP}}=1.95(1-x)$ для $x>0.4$, но в области составов $x \sim 0.4$ наблюдается сильный разрыв в распределении сил осцилляторов и для $x<0.4$ распределение сил осцилляторов становится $S_{\mathrm{GaP}}=1+0.95(1-x)$ с дополнительным увеличением сил осцилляторов $\Delta S_{\mathrm{GaP}} \sim 1.0 x$ в области составов $x<0.4$. Подобное поведение в распределении сил осциллятора наблюдается и для AІР-подобных колебаний, но для составов $x<0.4 S_{\text {AIP }}$ резко падает. В сплавах $\mathrm{Zn}_{1-x} \mathrm{Cd}_{x} \mathrm{Se}$ и $\mathrm{CdTe}_{1-x} \mathrm{Se}_{x}$ подобные скачки значений в композиционной зависимости сил осцилляторов наблюдаются для составов, соответствующих фазовому переходу между кубической (ZB) кристаллической структурой и гексагональной (W). На рис. 4 незаштрихованными символами показаны распределения сил осцилляторов $S_{\mathrm{GaP}}^{(-)}=S_{\mathrm{GaP}}-S_{\mathrm{GaP}}^{\mathrm{Al}}$ (с вычетом распределения сил осциллятора дополнительной моды $\mathrm{TO}_{\mathrm{GaP}}^{\mathrm{Al}}$ ) и $S_{\mathrm{AlP}}^{(+)}=S_{\mathrm{AlP}}+S_{\mathrm{GaP}}^{\mathrm{Al}}$ Функция $S_{\mathrm{GaP}}^{(-)}(x)$ становится практически линейной по всему составу сплава. Сглаживается и распределение $S_{\mathrm{AlP}}^{(+)}$Увеличение суммарной силы осцилляторов $\mathrm{GaP}$-подобных колебаний $\Delta S_{\mathrm{GaP}} \sim 1.0 x$ в области составов $x<0.4$ вызвано $\mathrm{Ga}-$ Р-колебаниями дополнительной моды $\mathrm{TO}_{\mathrm{GaP}}^{\mathrm{Al}}$ с увеличенной силой осциллятора в пересчете на единичную связь. В осциллятор-

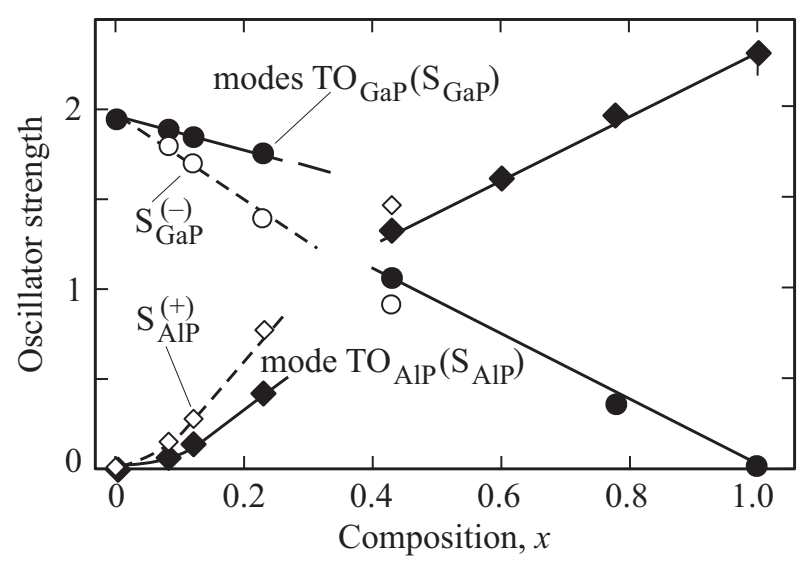

Рис. 4. Распределения суммарной силы осцилляторов $S_{\mathrm{GaP}}$ для всех $\mathrm{GaP-подобных} \mathrm{колебаний} \mathrm{и} \mathrm{силы} \mathrm{осциллятора} S_{\mathrm{AlP}}$ для AlP-подобных колебаний в зависимости от состава сплава $x$ (показано темными символами). Светлыми символами показаны распределения, если от $S_{\mathrm{GaP}}$ вычесть распределение силы осциллятора отщепленной $\mathrm{TO}_{\mathrm{GaP}}^{\mathrm{Al}}$ моды $\left(S_{\mathrm{GaP}}^{(-)}\right)$, а к распределению $S_{\mathrm{AlP}}-$ прибавить $\left(S_{\mathrm{AlP}}^{(+)}\right)$. 
ной модели ТО-колебаний в полярном полупроводнике, из которой получено дисперсионное выражение (1) для диэлектрической функции $\varepsilon(\omega)$, сила осциллятора для $j$-ой ТО-моды $S_{j}=\frac{e_{T j}^{2}}{\mu_{j} \omega_{t f}^{2}} \frac{N_{j}}{\varepsilon_{0}}$, где $\mu-$ приведенная масса ионной пары, $e_{T}-$ поперечный эффективный заряд, $N$ - количество ионных пар на единицу объема. Увеличенное значение суммарной силы осцилляторов $S_{\mathrm{GaP}}$ для GaP-подобных колебаний сопровождается появлением дополнительной моды GaP-подобных колебаний $\mathrm{TO}_{\mathrm{GaP}}^{\mathrm{Al}}$, в которой сила осциллятора в пересчете на единичную связь увеличивается за счет перераспределения заряда между $\mathrm{Al}-\mathrm{P}$-связью и $\mathrm{Ga}-\mathrm{P}-$-вязью. К дополнительному обсуждению этого вопроса вернемся позже.

Для идентификации природы неизвестных мод в области GaP-подобных колебаний мы провели расчет колебательного спектра примесного $\mathrm{Al}$ в кристалле GaP с использованием микроскопической теории динамики кристаллической решетки в приближении малой концентрации примеси [14]. В рамках этой теории рассматривается поведение локальной моды, возникающей при замещении атома Ga более легким атомом $\mathrm{Al}$ в кристалле $\mathrm{GaP}$ с функцией плотности однофононных состояний, в которой имеется щель с нулевой плотностью состояний в области частот $\mathrm{LO}-\mathrm{TO}-$-расщепления $\mathrm{Ga}-\mathrm{P}$ колебаний (рис. 2,a). При расчете вводится только один примесный параметр, дефект массы $\eta=m_{\mathrm{Al}} /\left(m_{\mathrm{Ga}}-m_{\mathrm{Al}}\right)$, при неизменном параметре кристаллической решетки. В соответствии с микроскопической теорией, диэлектрическая функция $\varepsilon(\omega)$ сплава $A_{1-x} B_{x} c$ при малом содержании одной из компонент $(x \ll 1)$ определяется из уравнений [14]

$$
\begin{gathered}
\varepsilon(\omega)=\varepsilon_{\infty}+\frac{\left(\varepsilon_{0}-\varepsilon_{\infty}\right) \omega_{\mathrm{TO}}^{2}}{\omega_{\mathrm{TO}}^{2}-\left(\omega^{2}+i \omega \gamma\right) \chi(x, \omega)}, \\
\chi(x, \omega)=\frac{(1-x) F\left(A, \omega^{2}\right)-\eta}{(1-x) F\left(A, \omega^{2}\right)-\eta-x \frac{m_{C}}{m_{A}+m_{C}}} .
\end{gathered}
$$

Здесь $\eta=m_{B} /\left(m_{B}-m_{A}\right)-$ массы соответствующих атомов, $\omega_{\text {Tо }}$ - частота ТО-колебаний беспримесного кристалла $A C$,

$$
F\left(A, \omega^{2}=-1+\omega^{2} G\left(A, \omega^{2}\right)\right.
$$

$G\left(A, \omega^{2}\right)-$ функция Грина для кристалла $A C$, в котором замещается атом $A$. Для сплава $\mathrm{Ga}_{1-x} \mathrm{Al}_{x} \mathrm{P}$ с малым содержанием Al функция Грина $G\left(\mathrm{Ga}, \omega^{2}\right)$ рассчитывалась с использованием функции плотности фононных состояний чистого GaP из [10], хотя для расчета необходимо знать парциальную плотность квадратов частот. Мы не нашли в литературе расчет для этой функции. При определенных условиях, например при близких значениях масс атомов в элементарной ячейке, такая замена вполне оправдана. Более подробное изложение микроскопической теории представлено в совместной с автором теории работе [15]. В рассчитанной диэлек- трической функции $\operatorname{Im} \varepsilon(\omega)$ (рис. $2, b)$ помимо основной решеточной моды $\mathrm{GaP}\left(\mathrm{c} \omega=365 \mathrm{~cm}^{-1}\right)$, априорно задаваемой при расчете, и локальной моды для малых значений $x$, которая с увеличением $x$ переходит в кристаллическую AlР-подобную ТО-моду, проявляется слабая мода с $\omega=378 \mathrm{~cm}^{-1}$, соответствующая по частоте нулевому провалу в однофононной плотности состояний $\mathrm{GaP}$ (рис. 2,a). Аналогичное отщепление слабой моды с $\omega=378 \mathrm{~cm}^{-1}$ от основной моды GaP наблюдается при расчете колебательного спектра примесного As в кристалле GaP. В отличие от предыдущего расчета $\operatorname{Im} \varepsilon(\omega)$ для $\mathrm{Ga}_{1-x} \mathrm{Al}_{x} \mathrm{P}$, в рассчитанной диэлектрической функции $\operatorname{Im} \varepsilon(\omega)$ сплава $\mathrm{GaP}_{1-y} \mathrm{As}_{y}$ для малых $y$ замещение атома $\mathrm{P}$ более тяжелым As индуцирует появление щелевой моды, переходящей с увеличением $y$ в кристаллическую GaAs-подобную ТО-моду, и моды $\mathrm{Ga}-\mathrm{P}$-колебаний оказываются высокочастотной полосой в сплаве $\mathrm{GaP}_{1-y} \mathrm{As}_{y}$. Ранее [16] подобная слабая мода на частоте, попадающей в щель с нулевой плотностью фононных состояний $\mathrm{ZnSe}$, наблюдалась и расчитывалась для II-VI сплавов $\mathrm{Zn}_{1-x} \mathrm{Cd}_{x} \mathrm{Se}$ и $\mathrm{ZnSe}_{1-x} \mathrm{Te}_{x}$ с малой концентрацией $\mathrm{Cd}$ и Те соответственно. И позже [15] дополнительная слабая мода в сплаве $\mathrm{Hg}_{1-x} \mathrm{Cd}_{x} \mathrm{Te}$, индуцированная $\mathrm{Cd}$-примесным замещением атома $\mathrm{Hg}$, рассчитывалась и наблюдалась на частоте, соответствующей сильному провалу в однофононной плотности состояний HgТе. Представленные выше расчеты колебательного спектра примесных атомов Al и As в кристалле $\mathrm{GaP}$ показывают, что дополнительная мода с $\omega=378 \mathrm{~cm}^{-1}$ не является дефектной модой, связанной с несоверешенством сплава или с неконтролируемым наличием примесей, а вызвана особенностями фононной системы сплава.

В перколяционной схеме (одна связь $\rightarrow$ две моды) перестройки колебательного спектра сплава с составом дополнительная мода с $\omega=378 \mathrm{~cm}^{-1}\left(\mathrm{TO}_{\mathrm{GaP}}^{\mathrm{Al}}\right)$,

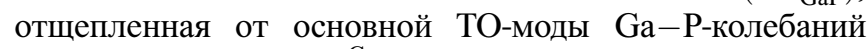
c $\omega=365 \mathrm{~cm}^{-1}\left(\mathrm{TO}_{\mathrm{GaP}}^{\mathrm{Ga}}\right)$, является одной из компонент перколяционного дуплета GaP-подобных колебаний. Pagès atal. [17], предлагая перколяционную схему перестройки колебательного спектра для сплава $\mathrm{GaP}_{1-y} \mathrm{As}_{y}$, рассматривают тот же перколяционный дуплет GaP-подобных колебаний с отщепленной от ТО-моды GaP дополнительной моды с $\omega=378 \mathrm{~cm}^{-1}$. Проявления перколяционного дуплета для $\mathrm{Ga}-\mathrm{As}-$ колебаний авторы не ожидают из-за большой дисперсии частоты ТО-колебаний $\left(\sim 15 \mathrm{~cm}^{-1}\right)$ в чистом GaAs кристалле. В соответствии с критерием Андерсона (the Ayderson's criterion), так называемая фононная локализация, т.е. регистрация нескольких ТО-мод колебаний на связь, проявляется, если флуктуация по частоте ТО-моды превышает ее дисперсию в соответствующем чистом кристалле [18]. В GaP дисперсия ТО-моды почти нулевая, смещаясь только на $\sim 1.5 \mathrm{~cm}^{-1}$ в зоне от центра до края $(X)$ зоны [17], тем самым допуская фононную локализацию для $\mathrm{GaP}-$ подобных колебаний с конечным расщеплением перколяционного дуплета. На- 
ши расчеты колебательного спектра примесных атомов в II-VI и III-V кристаллах, в которых в плотности фононных состояний между частотами TO- и LO-мод колебаний имеется щель с нулевой плотностью фононных состояний, указывают на наличие критерия проявления в спектре сплава перколяционного дуплета - если дополнительная мода по частоте попадает в щель с нулевой плотностью фононных состояний, она является компонентой перколяционного дуплета. Это утверждение не противоречит критерию Андерсона, т. к. наличие щели с нулевой плотностью фононных состояний между частотами TO- и LO-мод колебаний чистого кристалла указывает на малую дисперсию частоты ТО-колебаний в кристалле.

B [17] Pagès at al. рассматривают сплав $\mathrm{GaP}_{1-y} \mathrm{As}_{y}$ как композитную среду, образованную сосуществующими GaP- и GaAs-обогащенных областями. Две колебательные моды на связь возникают из-за различия длин связи, вызванного локальными искажениями связи в соответствии с тем, в какой области GaP-обогащенной или GaAs-обогащенной, рассматривается связь. В случае со сплавом $\mathrm{Ga}_{1-x} \mathrm{Al}_{x} \mathrm{P}$, в котором решеточные параметры бинарных компонент сплава почти совпадают, никакого изменения в длине связи не происходит, но перколяционный дуплет GaP-подобных колебаний с отщепленной от ТО-моды $\mathrm{GaP}$ дополнительной моды с $\omega=378 \mathrm{~cm}^{-1}$, наблюдается. Имеет смысл обратиться к старой кристаллической модели поля валентных сил VFF (valenceforce-field) [19] для тетраэдрически координированных кристаллов, в которой все межатомные силы разделяются на центральные (bond-stretching) и нецентральные (bond-bending) силы. В этом приближении все искажения кристаллической решетки описываются через скалярные изменения длины связи $(\Delta r)$ и угла между связями $(\Delta \theta)$ соответственно. Для расчетов упругих свойств кристалла и представления их результатов используются константы валентных сил $\alpha$ и $\beta$, которые соотносятся с силовыми константами центральных и нецентральных сил

$$
k_{r}=3 \alpha+\beta / 2 \text { и } k_{\theta}=2 \beta / 3 .
$$

В таблице представлены значения длин связи (в $\AA$ ) и констант валентных сил $\alpha$ и $\beta(\mathrm{N} / \mathrm{m})$ для некоторых соединений [20]. Прослеживается общая тенденция к уменьшению константы $\beta$ по мере увеличения ионности связи $f_{r}$. В [19] было найдено эмпирическое соотношение $\beta / \alpha \sim 1-f_{i}$, из которого следует $\beta=0$ при $f_{i}=1$ (для ионного кристалла). Параметр $\beta$ для GaP из общей тенденции сильно выделяется, он близок к $\mathrm{Si}$ и совпадает c Ge. чисто ковалентными кристаллами. Для пары GaAs и AlAs - бинарных компонент сплава GaAlAs - параметры валентных сил $\alpha$ и $\beta$ почти совпадают (в пределах 5\%). Это означает, что при образовании сплава никаких существенных деформаций связей не происходит, в колебательном спектре сплава проявляются только GaAs- и AlAs-подобные моды колебаний. Для пары GaP и AlP - бинарных компонент
Длины связи $d$ (в $\AA$ ) и параметры валентных сил $\alpha$ и $\beta(\mathrm{N} / \mathrm{m})$

\begin{tabular}{c|c|c|c}
\hline Compound & $d$ & $\alpha$ & $\beta$ \\
\hline $\mathrm{Si}$ & 2.352 & 48.50 & 13.81 \\
$\mathrm{Ge}$ & 2.450 & 38.67 & 11.35 \\
$\mathrm{AlP}$ & 2.367 & 44.323 & 8.068 \\
$\mathrm{AlAs}$ & 2.451 & 40.849 & 8.717 \\
$\mathrm{GaP}$ & 2.360 & 44.764 & 10.737 \\
$\mathrm{GaAs}$ & 2.448 & 39.235 & 9.159 \\
$\mathrm{CdTe}$ & 2.806 & 26.569 & 2.722 \\
$\mathrm{HgTe}$ & 2.798 & 26.396 & 2.746
\end{tabular}

сплава GaAlP - соотношение параметров валентных сил иное, при равенстве длин связи разница в значениях параметра $\beta$ (параметра нецентральных сил) достигает $30 \%$. В GaP очень высокая степень ковалентности связи. Ранее [21], при исследовании аномальных свойств решеточных колебаний $\mathrm{HgTe}$ в сплаве $\mathrm{HgCdTe}$ мы встречались с фотоэмиссионными исследованиями электронной структуры матрицы $\mathrm{HgTe}$ при введении в нее Mn и

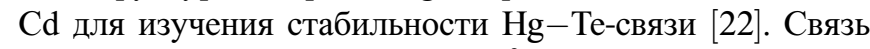
$\mathrm{Cd}-\mathrm{Te}$ осуществляется через $s p^{3}$-орбитали, а в $\mathrm{HgTe}-$ связь тяжелой $\mathrm{Hg}$ с Те в значительной степени формируется с участием $d$-орбиталей [6,7] и в меньшей степени через $s p^{3}$-орбитали. Из расчетов соответствующих „химических сдвигов“ установлена сильная дестабилизация $\mathrm{Hg}-$ Те-связи за счет того, что сильная $\mathrm{Cd}-\mathrm{Te} s p^{3}$-связь оттягивает Те $p$-орбиталь от более слабой $\mathrm{Hg}-\mathrm{Te}-$-вязи. Если допустить, что в сплаве $\mathrm{GaAlP} \mathrm{Ga}-\mathrm{P} s p^{3}$-связь значительно сильнее $\mathrm{Al}-\mathrm{P} s p^{3}$-связи в соответствии со значениями параметра $\beta$, то она может стать более жесткой в AlP-обогащенной области сплава за счет оттягивания анионной Р p--орбитали, которое будет сопровождаться перераспределением заряда между Al-P- и

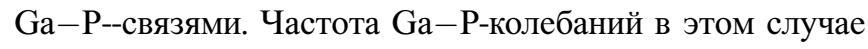
увеличится (для дополнительной моды), увеличится и сила осциллятора дополнительной моды в пересчете на единичную связь.

В спектре решеточных колебаний сплава $\mathrm{Ga}_{1-x} \mathrm{Al}_{x} \mathrm{P}$ в области частот $\mathrm{Ga}-\mathrm{P}$-подобных колебаний проявляется еще одна мода $\mathrm{TO}_{\mathrm{GaP}}^{\mathrm{add}}$ на частоте $355 \mathrm{~cm}$ (рис. 2,c), производная от щелевой моды AlP : Ga при $x \rightarrow 1$ (рис. 3,a). С подобной многомодовостью мы сталкивались при интерпретации колебательных спектров сплава HgCdTe (например, [5]), в котором решеточное рассогласование между бинарными компонентами сплава практически отсутствует, как и в GaAIP. По аналогии c $\mathrm{HgCdTe}$, расщепление моды GaР-подобных колебаний в сплаве GaAlP интерпретируется в квазимолекулярном приближении [23], в соответствии с которым сплав рассматривается образованным пятью базисными ячейками $\mathrm{Ga}(4-n) \mathrm{Al}(n) \mathrm{P}(n=0,1,2,3,4)$ из катионов $\mathrm{Ga}$ и $\mathrm{Al}$, тетраэдрически расположенных вокруг общего аниона P. Каждой базисной ячейке соответствует своя мода $\mathrm{Ga}-\mathrm{P}$ и $\mathrm{Al}-\mathrm{P}$-колебаний, и спектр решеточных колебаний 
сплава апроксимируется свойствами четырех мод $\mathrm{Ga}-\mathrm{P}-$ колебаний с ${ }^{\mathrm{GA}} \omega_{n}(n=0,1,2,3)$ и четырех мод $\mathrm{Al}-\mathrm{P}$ колебаний с ${ }^{\mathrm{Al}} \omega_{n}(n=1,2,3,4)$ пяти базисных ячеек $\mathrm{Ga}(4-n) \mathrm{Al}(n) \mathrm{P}(n=0,1,2,3,4)$ через соответствующее распределение вероятностей $P_{n}(x)$ их различных конфигураций в сплаве $\mathrm{Ga}_{1-x} \mathrm{Al}_{x} \mathrm{P}$ разного состава. В реальном спектре сплава конкретного состава все моды проявляться не могут, наблюдаются наиболее сильные. Расщепления AlР-подобных мод не наблюдалось из-за большой дисперсии частоты ТО-колебаний в AlP. B GaP дисперсия ТО-моды колебаний почти нулевая, около $1.5 \mathrm{~cm}^{-1}$, тем самым допуская фононную локализацию (т.е. многомодовость) для $\mathrm{GaP-подобных} \mathrm{колебаний.} \mathrm{Расчетные}$ кривые для сил осциллятора ${ }^{\mathrm{Ga}} S_{n}(x) n$-ой моды GaPколебаний определяются выражением [5]

$$
{ }^{\mathrm{Ga}} S_{n}(x)={ }^{\mathrm{Ga}} f_{n} N \frac{4-n}{4} \frac{4 !}{n !(4-n) !}(1-x)^{4-n} x^{n},
$$

где ${ }^{\mathrm{Ga}} f_{n}-$ приведенная к единичной связи $\mathrm{Ga}-\mathrm{P}$ сила осциллятора. Аппроксимирующая кривая для композиционной зависимости сил осциллятора GaP-подобной моды $\mathrm{TO}_{\mathrm{GaP}}^{\mathrm{Ga}}$ на рис. $3, b$ описывается расчетными функциями ${ }^{\mathrm{Ga}} S_{n}(x)$ для $n=0$ и $1,{ }^{\mathrm{Ga}} S_{0}(x)+{ }^{\mathrm{Ga}} S_{1}(x)=$ $=1.95\left[(1-x)^{4}+3 x(1-x)^{3}\right]$. Композиционная зависимость сил осциллятора дополнительной $\mathrm{GaP}$-подобной моды $\mathrm{TO}_{\mathrm{GaP}}^{\mathrm{add}}$ описывается функциями ${ }^{\mathrm{Ga}} S_{n}(x)$ для $n=2$ и $3,{ }^{\mathrm{Ga}} S_{2}(x)+{ }^{\mathrm{Ga}} S_{3}(x)$. Многомодовое расщепление в сплаве вызвано диполь-дипольным взаимодействием между ближайшими ионами, связанным с многообразием локального окружения общего аниона $\mathrm{P}$ катионами Ga и Al. Из-за различия масс катионов их смещения (амплитуды колебаний) и соответствующие дипольные моменты, индуцированные макроскопическим полем, будут различными, что и приводит к различным частотам мод для разных катионных конфигураций $(n=0-5)$ вокруг общего аниона в сплаве. Дополнительной причиной проявления многомодовости в сплаве GaAlP является большое различие (в соответствии с валентным параметром $\beta$ ) в сдвиговой жесткости $\mathrm{Ga}-\mathrm{P}-$ и $\mathrm{Al}-\mathrm{P}-$ связей. $\mathrm{B}$ сплаве $\mathrm{HgCdTe}$ - различие в природе химической связи компонентов сплава. А проявится ли многомодовость в спектре сплава GaAlP, зависит в соответствии с критерием фононной локализации Андерсона от дисперсии ТО-моды в чистом GaP. Она очень низкая, $\sim 1.5 \mathrm{~cm}^{-1}$. Дополнительная мода $\mathrm{TO}_{\mathrm{GaP}}^{\mathrm{add}}$ является модой $\mathrm{GaP}$-подобных колебаний в сплаве GaAlP, обогащенном $\operatorname{AlP}(x>0.5)$.

\section{3. Заключение}

Спектр решеточных колебаний сплава $\mathrm{Ga}_{1-x} \mathrm{Al}_{x} \mathrm{P}$ в области GaP-подобных колебаний характеризуется (рис. 3,a) тремя модами колебаний. Расчет спектра примесного атома Al в кристалле GaP с использованием микроскопической теории динамики кристаллической решетки в приближении малой концентрации примеси показал, что помимо основной решеточной моды $\mathrm{TO}_{\mathrm{GaP}}^{\mathrm{Ga}}\left(\right.$ с $\left.\omega=365 \mathrm{~cm}^{-1}\right)$, априорно задаваемой при расчете, и локальной моды для малых значений $x$, которая с увеличением $x$ переходит в кристаллическую AlP-подобную $\mathrm{TO}_{\mathrm{Al}}$ моду, проявляется слабая мода с $\omega=378 \mathrm{~cm}^{-1}$, по частоте совпадающая с нулевым провалом в однофононной плотности состояний кристалла $\mathrm{GaP}$ (рис. 2,a,b). В перколяционной схеме перестройки колебательного спектра сплава $\mathrm{Ga}_{1-x} \mathrm{Al}_{x} \mathrm{P}$ с составом $x$ дополнительная мода на частоте $\omega=378 \mathrm{~cm}^{-1}$, отщепленная от основной $\mathrm{GaP}$ моды, является следствием проявления композитного состава сплава. Мода $\mathrm{TO}_{\mathrm{Ga}}^{\mathrm{Al}}$ с $\omega=378 \mathrm{~cm}^{-1}$ является производной от основной моды $\mathrm{Ga}-\mathrm{P}$-колебаний и одной из компонент перколяционного дуплета. Область перколяционного расщепления ограничивается составом сплава с $x \leq 0.4$ и сопровождается структурной модификацией, связанной с переносом заряда между $\mathrm{Ga}-\mathrm{P}$ и Al-P-связями. Сила осциллятора моды решеточных колебаний пропорциональна $e_{T}^{2}\left(e_{T}-\right.$ поперечный эффективный заряд связи). При анализе композиционного распределения сил осцилляторов GaР-подобных и AlPподобных колебаний наблюдается скачек в распределениях: при $x<0.4$ суммарная сила осцилляторов $\mathrm{GaP}$ подобных колебаний резко увеливается, а AlP-подобных колебаний - в такой же степени уменьшается. Мода $\mathrm{TO}_{\mathrm{GaP}}^{\mathrm{add}}$ проявляется в сплаве $\mathrm{Ga}_{1-x} \mathrm{Al}_{x} \mathrm{P}$, обогащенном AlP $(x>0.5)$, и является модой GaP-подобных колебаний для составов, в которых преимущественными структурными элементами считаются базисные ячейки $\mathrm{Ga}(4-n) \mathrm{Al}(n) \mathrm{P}(n=2$ и 3$)$ из катионов $\mathrm{Ga}$ и $\mathrm{Al}$, тетраэдрически расположенных вокруг общего аниона P.

\section{Список литературы}

[1] D. Stauffer. Introduction to Percolation Theory. Taylor and Francis, London (1985).

[2] O. Pagès, M. Ajjoun, D. Bormann, C. Chauvet, E. Tourniè, J.P. Faurie. Phys. Rev. B 65, 035213 (2002).

[3] O. Pagès, T. Tite, K. Kim, P.A. Graf, O. Maksimov, M.C. Tamargo. J. Phys.: Condens. Matter 18, 577 (2006).

[4] С.П. Козырев. ФТТ 57, 1790 (2015).

[5] С.П. Козырев. ФТТ 54, 1743 (2012).

[6] S.H. Wei, A. Zunger. Phys. Rev. B 37, 8958 (1988).

[7] Duan He, Dong You-Zhong, Huang Yan, Chen Xiao-Shuang. Chin. Phys. B 20, 043103 (2011).

[8] G. Lukovsky, R.D. Burnham, A.S. Alimonda. Phys. Rev. B 14, 2503 (1976).

[9] O.K. Kim, W.G. Spitzer. Phys. Rev. J. Appl. Phys. 50, 4362 (1979).

[10] K. Kunc, M. Balkanski, M.A. Nasimovici. Phys. Status Solidi B 72, 229 (1975).

[11] P.N. Sen, G. Lukovsky. Phys. Rev. B 12, 2998 (1975). 
[12] G. Armelles, J.N. Calleja, E. Munoz. Solid State Commun. 65, 779 (1988)

[13] Л.К Водопьянов, С.П. Козырев, Н.Н. Мельник. ФТТ 42 , 618 (2000).

[14] В.С. Виноградов. ФТТ 11, 2062 (1969).

[15] С.П. Козырев, В.С. Виноградов. ФТТ 55, 375 (2013).

[16] С.П. Козырев. ФТП 48, 1297 (2014).

[17] O. Pagès, J. Souhabi, A.V. Postnikov, A. Chafi. Phys. Rev. B 80, 035204 (2009).

[18] M. Cardona, P. Etchegoin, H.D. Fuchs, P. Molina-Mata. J. Phys.: Condens. Matter 5, A61 (1993).

[19] R.M. Martin. Phys. Rev. B 1, 4005 (1970).

[20] A.-B. Chen, A. Sher. Phys. Rev. B 32, 3695 (1985),

[21] С.П. Козырев. ФТТ 53, 153 (2011).

[22] A. Wall, C. Capnele, A. Franciosi, R. Reifenberger, U. Debska. J. Vac. Sci. Technol. A 4, 818 (1986).

[23] H.W. Verleur, A.S. Barker. Phys. Rev. 149, 715 (1966). 